Ann. Biol. anim. Bioch. Biophys., I 955,5 (I), I5 I-I56.

\title{
DUPLICATION DE L'ADN DANS LES OEUFS DE LAPINE APRÈS LA FÉCONDATION
}

\author{
St. OPRESCU $\left({ }^{1}\right)$ et $r$. THIBAULT \\ avec la collaboration technique de Anne-Marie ZIMMERMANX \\ Station centrale de Physiologie animale, \\ Centre national de Recherches zootechniques, Jouv-en-Josas (Seine-et-Oise)
}

SOMMAIRE

Des oufs fécondés de Lapine ont été cultivés de 1 à 3 heures en présence de thymidine tritiée à des périodes comprises entre l'ovulation et la fin de la première division de segmentation.

La duplication de l'ADN s'effectue essentiellement au moment où les pronuclei pleinement formés se rapprochent l'un de l'autre (stade IV); il s'écoule donc environ 3 heures entre la fécondation et le marquage (phase $G_{1}$ ), celui-ci ( $S$ ) dure une à deux heures et la première division ne se produira que 4 à 5 heures plus tard (phase $G_{2}$ ). Au cycle cellulaire suivant, la phase $G_{1}$ disparait presque complètement et la période de synthèse $\mathrm{S}$ reste également de l'ordre de i heure.

\section{INTRODUCTION}

Chez les Mammifères, SIRLIx et EdDWARDs (I959), utilisant de l'adénine ${ }^{14} \mathrm{C}$ ont observé le marquage des pronuclei seulement I3 heures après la fécondation, c'està-dire peu de temps avant la première division de segmentation. En outre, le pronucleus mâle se marquerait le premier. Mais leurs images sont peu nettes et le stade exact des pronuclei n'est pas précisable. Ils ne fournissent enfin aucune information sur la durée de synthèse.

Chez les Invertébrés, l'étude faite sur l'Oursin (Srmmel, KARxorsky, I96I ; Hinegardier, FELDMAN, Ig64) montre une duplication rapide et précoce de l'ADN dans les pronuclei. La durée de synthèse est beaucoup plus brève que les

(1) Adresse actuelle : Institut de Biologie "Tr. Sävulescu ", Laboratoire de Génétique animale, Bucarest, Roumanie. 
durées généralement admises et qui varient de 3 à $\delta$ heures, ce qui s'explique par la rapidité du rythme des divisions.

Nous avons étudié chez la Lapine la duplication de 1'ADN depuis la fécondation jusqu'à la première division.

\section{MATÉRIEL ETT MÉTHODES}

Vingt-neuf Japines élevées depuis plusieurs mois an laboratoire ont été accouplées avec des mâles fertiles.

Elles ont été abattues I 2 à 23 heures après l'accouplement. Les aufs ont été recueillis par perfusion avec du liquicle de Locke et mis en culture pendant une à trois heures en présence de thymidine tritiée à raison d'une $\mu \mathrm{Ci}$ par $\mathrm{ml}$ de Locke. Les cuufs ont été généralement fixés immédiatement après l'incubation avec la thymidine, quelquefois cultivés pendant I à 6 heures supplémentaires dans du Locke ou dans du sérum homologue.

Le Bouin IIollande a été le fixateur employé et les techniques d'inclusion et de coupes furent celles décrites antérieurement (THIBALLT, 1949).

Après déparaffinage, les coupes à to $u$ ont été étalées sur des lames spéciales Ilford, puis recouvertes de l'émulsion pelliculable $\mathrm{Kodak} \mathrm{AR}$ ro et maintenues I mois à $+4^{\circ} \mathrm{C}$ dans une enceinte en plomb. Après révélation dans l'amidol (chlorhydrate de diamidophénol) ct fixation, les lames étaient plongées 25 à 30 minutes dans une solution de rouge solide Merck à 0 , I p. I00 (I g/litre dans une solution de $\left(\mathrm{SO}_{4}\right)_{3} \mathrm{Al}_{2} \dot{a}_{5} 5 \mathrm{p}$. 10o). Cette coloration permet un excellent repérage des ocufs sur les lames et une analyse suffisante de l'état nucléaire. La chromatine et les nucléoles apparaissent en rouge plus soutenu que le fond du cytoplasme; de plus, les gros pronuclei apparaissent comme des taches claires piquetées seulement par la chromatine ou les nucléoles. En cas d'hésitation sur l'état nucléaire, l'examen au contraste de phase permettait de le préciser.

\section{RÉSULTATS}

L'ensemble des expériences et des résultats est résumé dans les tableaux I et 2.

L'analyse de l'état nucléaire des oufs est indispensable pour permettre une interprétation correcte du moment du marquage ; on ne peut se référer au délai écoulé depuis l'accouplement que pour une estimation grossière du moment du marquage, car la pénétration du spermatozoïde ne se produit pas dans tous les œufs d'une même ponte au mêtne moment et il existe des différences dans le délai de fécondation d'une lapine à l'autre. En outre, les conditions de culture dans le Locke n'autorisent pas à admettre que l'évolution des pronuclei dans ce milieu se soit effectuée à une vitesse normale (Ex. lapine 67).

En se référant aux stades de la fécondation che $z$ la Lapine décrits par 'Thrbaurit (I965), on doit distinguer les stades suivants :

Stade II: CEufs venant d'être fécondés : le spermatozoïde fécondant occupe encore une position tout à fait nuarginale, le $2^{\mathrm{e}}$ globule polaire étant ou non complètement émis.

Stade III : Guts dans lesquels le pronucleus mâle formé est encore de petite taille, tandis que le pronucleus femelle est seulement en formation. A ce stade, un puissant aster mâle apparaît.

Stade IV : Migration des pronuclei l'un vers l'autre ; l'aster mâle est encore bien visible et de nombreux nucléoles existent dans les pronuclei. 
Stade $V$ : Les pronuclei sont centraux et accolés, ils ne renferment que 2 à $3 \mathrm{nu}-$ cléoles.

Sur 28 œufs provenant de 6 lapines et se trouvant au stade II à la fin de l'incubation avec la thymidine, aucun n'a été marqué. Il en fut de même sur 2 I œufs provenant de 6 lapines et se trouvant au stade III.

TABIEAU I

Durée de mise en incubation avec la thymidine tritice

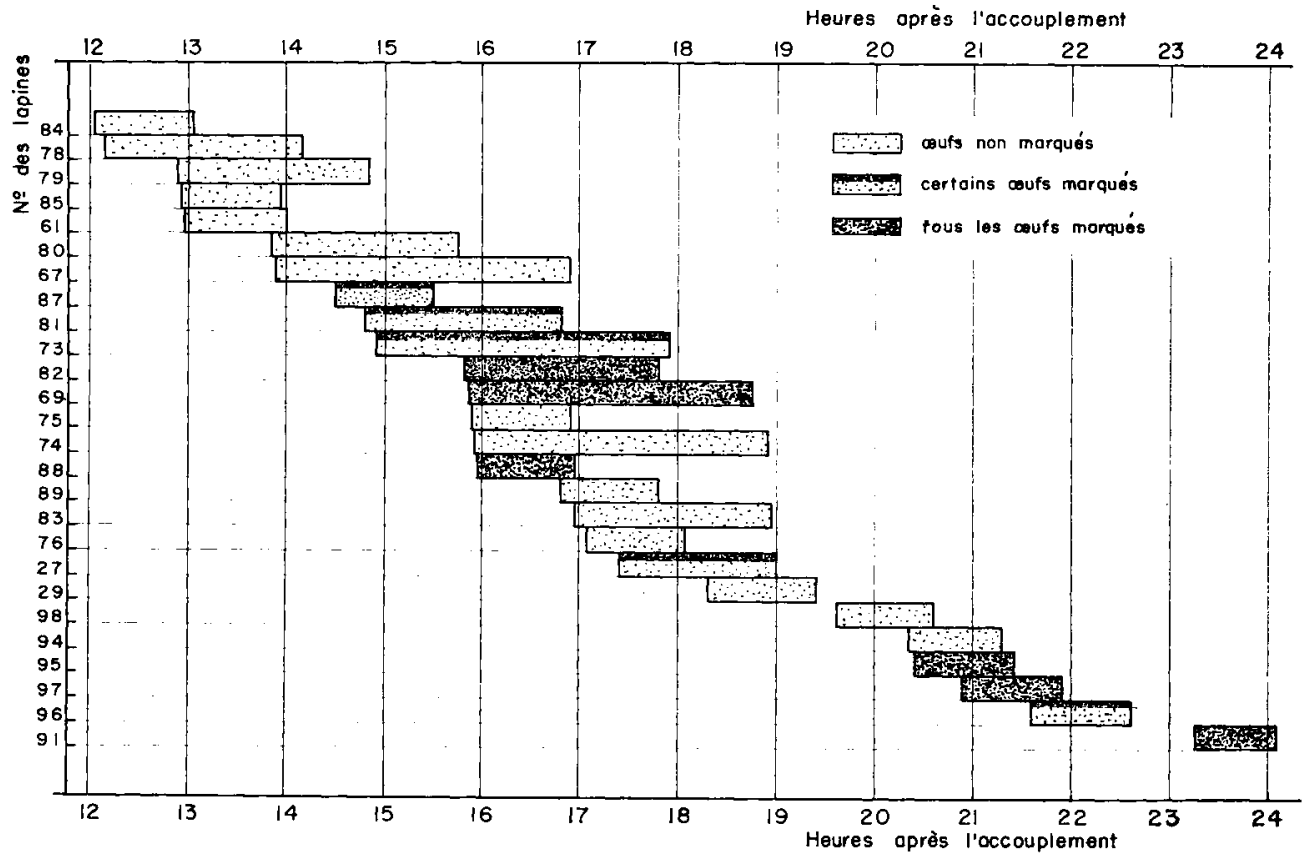

Au contraire, sur I 9 oufs provenant de 7 lapines et se trouvant au stade IV, tous étaient marqués.

Sur 36 œufs provenant de ro lapines se trouvant aul stade $V$, seulement 9 étaient marqués.

Sur 6 oufs provenant de 2 lapines et mis en contact avec la thymidine au moment de la prophase de la première division de segmentation, aucun n'était marqué.

II œufs provenant de 3 lapines et incubés I heure avec de la thymidine juste après la segmentation étaient tous marqués.

On remarque donc que la duplication de 1 'ADN ne se produit qu'à partir du stade IV et qu'elle est pratiquement limitée à ce stade. En effet, seulement 9 sur 36 œufs ont été trouvés marqués au stade $V$.

De plus, sur ces 9 œufs :

- 4 provenaient d'une lapine dont les autres œufs (8) n'étaient pas fécondés (lapine 97), ce qui suggère une fécondation tardive des quatre œufs fécondés ; 
- 3 provenaient d'une lapine dont 5 autres œufs marqués étaient au stade IV (lapine 88).

La fécondation se produit en moyenne I2 heures après l'accouplement, soit environ une heure trente après le début de l'ovulation (voir ThrBaulit, I965) (1). I a duplication de 1'ADN débute environ 3 heures après la fécondation. Sa durée ne paraît pas dépasser 2 heures (durée du stade IV ; marquage aussi intense après une heure de contact avec la thymidine qu'après deux ou trois heures).

La première division de segmentation se produit environ quatre heures après la duplication de l'ADN.

TABLEAU 2

\begin{tabular}{|c|c|c|c|c|c|c|c|}
\hline & \multicolumn{6}{|c|}{ Stade des œufs } \\
\hline Lapine No & $\begin{array}{l}\text { Eufs non } \\
\text { fécondés }\end{array}$ & Stade 2 & Stade 3 & Stade 4 & Stade 5 & $\begin{array}{l}\text { Prophase ou } \\
\text { Métaphase } \\
\text { de la 1 ere } \\
\text { division }\end{array}$ & Segmentés \\
\hline $\begin{array}{l}84 \\
78 \\
79 \\
85 \\
61 \\
80 \\
67 \\
87 \\
81 \\
73 \\
82 \\
69 \\
75 \\
74 \\
88 \\
89 \\
83 \\
76 \\
27 \\
29 \\
98 \\
94 \\
95 \\
97 \\
96 \\
91\end{array}$ & $\begin{array}{l}8 \\
1\end{array}$ & $\begin{array}{l}\text { N. M. (5) } \\
\text { N. H. (5) } \\
\text { N. M. (9) } \\
\text { N. M. (4) } \\
\text { N. M. (1) } \\
\text { N. M. (4) }\end{array}$ & $\begin{array}{l}\text { N. M. (2) } \\
\text { N. M. (10) } \\
\text { N. Y. (2) } \\
\text { N. M. (1) } \\
\text { N. M. (1) } \\
\text { N. Y (5) }\end{array}$ & $\begin{array}{l}\text { M. (3) } \\
\text { M. (1) } \\
\text { M. (1) } \\
\text { M. (5) } \\
\text { M. (1) } \\
\text { M. (5) } \\
\text { M. (3) }\end{array}$ & \begin{tabular}{|} 
I. M. (2) \\
II. (1) \\
M. (3) \\
N. M. (7) \\
N. M. (4) \\
M. (1) + N. M. (2) \\
N. M. (4) \\
N. M. (7) \\
M. (4) \\
N. M. (1)
\end{tabular} & $\begin{array}{l}\text { N. M. (2) } \\
\text { N. M. (4) }\end{array}$ & $\begin{array}{l}\text { M. (1) } \\
\text { M. (1) } \\
\text { M. (9) }\end{array}$ \\
\hline
\end{tabular}

N. M. - Euf non marqué

M. - Euf marqué

(5) - Indique le nombre examinés après coupe, développement et coloration.

(I) Certains œufs sont pénćtrés dès le début de l'ovulation, soit to heures $30 \mathrm{mn}$ après l'accouplement; on les retrouve au stade IV, 2 heures $30 \mathrm{~mm}$ a 3 heures plus tard (TinBavlt, I $6_{5}$ ).

Les dernières pénétrations observées naturellement ont eu lieu trois à quatre heures après l'ovulation, on retrouve ces cufs au stade IV, trois heures plus tard. C'est peut-être le cas des oufs de la lapine 27. 
Si l'on assimile la période s'étendant de la fécondation à la première division à un cycle cellulaire, on peut dire que $G_{1}$ dure 3 heures à 4 heures, $S, 2$ heures ou moins et $\mathrm{G}_{2}, 4$ à 5 heures.

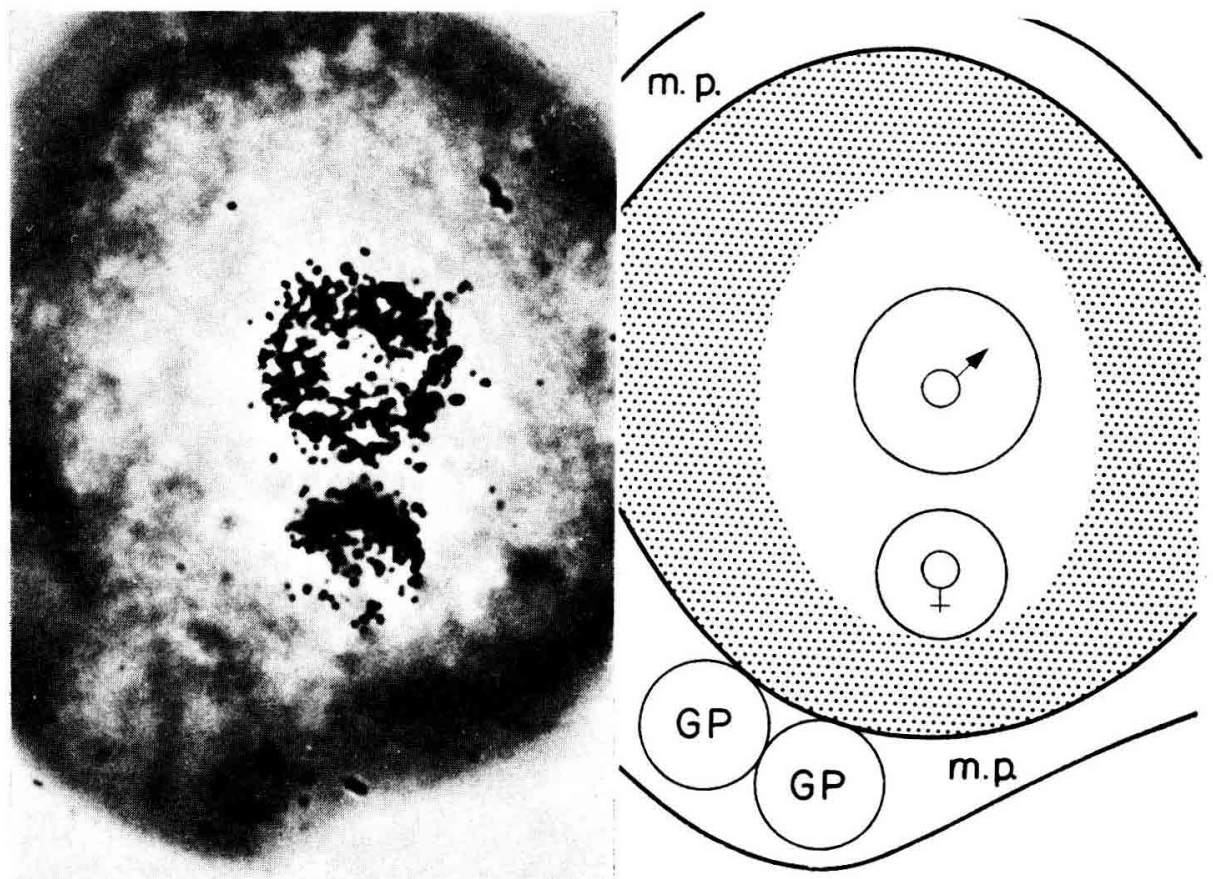

Euf narqué au stade IV :

Les deux pronuclëi $(q, \sigma)$ ne sont pas encore an contact et il subsiste un reste astérien indiqué par la zone claire entourant les pronucléi. Le pronucleus $q$ présente une distribution asymétrique de son $A D N$ (Thiballt, i 959), la majeure partie de celui-ci étant toumée vers le pronucleus mâle.

(r. P. : globules polaires

m. $\mathrm{p}:$ membrane pellucide

Au contraire, au cycle suivant, la phase $G_{1}$ est très brève, car la duplication de l'AION se fait immédiatement après la reconstitution des noyaux, elle paraît également s'effectuer en un temps court de l'ordre d'une heure.

\section{DISCUSSION ET CONCLUSIONS}

La duplication de l'ADN dans l'ouf de Lapine après la fécondation met en évidence une phase $G_{1}$ relativement courte au cours du premier cycle et presque inexistante au cours du second; la durée de la synthèse de l'ADN est beaucoup plus brève qu'on ne l'admet pour les tissus des Mammifères. Le déclenchement de la division n'est done pas lié à l'achèvement de la duplication de l'ADN, mais à l'établissement d'un certain état nucléaire ou cytoplasmique acquis au cours de la phase $\mathrm{G}_{1}$.

Les résultats récents de Szoli.osi (I964) diffèrent également des nôtres puisqu'à 
l'opposé de SIRIIN et EDwaris, il estime que la duplication de l'ADN commence peu après la pénétration du spermatozoïde chez le Lapin et le Hamster. Cet auteur considère également que la durée de synthèse est longue, de l'ordre de 5 à 6 heures, mais les méthodes d'estimation ne sont pas précisées.

Les observations de Simmer et Karnofsky (Ig6I) et Hinegardner et FeidD$\operatorname{MAN}(\mathrm{I} 964)$ chez l'Oursin sont au contraire en plein accord avec les nôtres : la synthèse de I'AIN se fait dans les pronuclei avant leur accolement.

I) plus, après la première division la synthèse a lieu si précocement, entre la télophase et l'interphase que HINEGARDNER et FEILMAN ont estimé que $\mathrm{G}_{1}$ disparaissait complètement.

I)ans deux cas, le pronucleus femelle (jeune) était seul très faiblement marqué, le cas inverse n'a pas été observé. SIRLIX et Fiwards ont, au contraire, estimé que le pronucleus mâle se marquait le premier. Quoiqu'il en soit, cette asynchronie est faible et probablement sans importance. On doit plutot remarquer que la duplication de 1'ADN s'effectue simultanément dans deux noyaux d'origine différente, non au contact, mais placés dans une unique cellule, ce qui implique une régulation par le cytoplasme.

L'origine des deux pronuclei est facilement identifiable chez la Lapine (THIBAUI, $T$, I965) et le marquage par la thymidine confirme la distribution asymétrique de l'ADN dans le pronucleus femelle.

$$
\text { Reçu pour publication en janvier } 1^{6} 65 \text {. }
$$

\section{SUMMARY}

\section{DUPLICATION OE DNA IN THE EGG OF THE RAPIBIT AFTER FERTILISATION}

The fertilised eggs of 29 rabbits were cultured for $I$ to 3 hours in the presence of tritiated thymidine from 12 to 23 hours after mating, After fixation and double inclusion the eggs were cut at to $\mu$ and covered with Kodak $\Lambda \mathrm{R}$ to photographic emulsion.

One month later the slides were examined after staining the eggs with solid red. This study showed that the duplication of DNA is short, from I to 2 hours, that it occurs about 3 hours after fertilisation and 4 to 5 hours before the first division of segmentation. After the first division the $G_{1}$ phase is very short and the synthesis begins immediately with the reconstitution of the nucleus; it also lasts only for $\mathrm{I}$ to 2 hours.

\section{RÉFÉRENCES BIBIIOGRAPHIQUES}

Glemfant S., igfiz. Initiation of mitosis in relation to the cell division cycle. Exp. Cell. Res., 26, 3I5-403. Hinegarderer R. T., Febmuax D. E., ig64. The DNA synthetic period during early development of the sea urchin egrr. Exp. Cell. Res., 36, 53-6r.

Post J., Hung ( Y., Howlinan J., 1963. The replication time and pattern of the liver cell in the growing Rat. I. Cell. Biol., 18, I-t2.

Srymel F. B., Kirvorshy D. A., I96I. Observations on the uptake of tritiated thymidine in the pronuclei of fertilized sand dollar embryos. /. Biophys. Biochem. Cytol., 10, 59-65.

Sirltix J. L., Euwards R. G., 1959. Timing of DNA synthesis in ovarian oocyte nuclei and pronuclei of the Mouse. Jixp. Cell. Res., 18, Ig0-194.

Szollosi D., I964. 'Time of DNA synthesis in mammalian eggs after sperm penetration (Abstr). J. Cell. Biol., 23, 92 A.

Thibaui, C., 1949. L'ouf des Nammiferes et son développement parthénogénétique. Ann. Sci. Nat., 11, I 36-2 I 1 .

Thibault C., I965. La fécondation. In Grassé Traité de Zoologie. Les Mammijères. Masson éd., à paraître. Zosmovslial A. I., ig63. The mitotic cycle in cells of mice studied with aid of the methor of radioautography (Russe). Dokl. Hkat. Nauk, 151, 687-6yo. 